\title{
OSCILLATION THEOREMS FOR NONLINEAR SECOND ORDER DIFFERENTIAL EQUATIONS WITH DAMPED TERM
}

\author{
CHEH-CHIH YEH
}

\begin{abstract}
Some new integral criteria for the oscillation of the nonlinear second order differential equation with damped term $y^{\prime \prime}(t)+p(t) y^{\prime}(t)+q(t) f(y(t))=0$ are given.
\end{abstract}

1. Introduction. Consider the linear differential equation

$$
y^{\prime \prime}(t)+a(t) y(t)=0
$$

where $a(t) \in C\left[t_{0}, \infty\right)$. By the well-known theorem of Wintner [12]

$$
\lim _{t \rightarrow \infty} \frac{1}{t} \int_{t_{0}}^{t} d u \int_{t_{0}}^{u} a(s) d s=\infty
$$

is sufficient for equation (1) to be oscillatory even when $a(t)$ is not assumed positive. Hartman [5] proved that the limit cannot be replaced by the upper limit in condition (2). In [6], Kamenev extended Wintner's result by using the $n$th primitive

$$
A_{n}(t)=\frac{1}{n !} \int_{t_{0}}^{t}(t-u)^{n-1} a(u) d u
$$

of the coefficient $a(t)$ for some integer $n \geqslant 3$.

Let $R$ be the set of all real numbers. Considering the nonlinear differential equation

$$
y^{\prime \prime}(t)+q(t) f(y(t))=0
$$

where $q \in C\left[t_{0}, \infty\right), f \in C(R), y f(y)>0$ for $y \neq 0$ and $f^{\prime}(y) \geqslant 0$ for all $y \in R$. Under the assumption that $q(t)$ is eventually nonnegative, Waltman [11] proved the following extension of an oscillatory result of Atkinson [1], who considered the special case $f(y)=y^{2 n+1}, n=1,2, \ldots$.

THEOREM A. Assume that for some $p>1, f(y)$ satisfies

$$
\liminf _{y \rightarrow \infty} \frac{f(y)}{|y|^{p}}>0 \text {. }
$$

Then a necessary and sufficient condition that all solutions of (3) are oscillatory is that

$$
\int^{\infty} t q(t) d t=\infty
$$

Received by the editors August 5, 1980.

AMS (MOS) subject classifications (1970). Primary 34K 15.

Key words and phrases. Differential equation with damped term, oscillation.

(C) 1982 American Mathematical Society $0002-9939 / 81 / 0000-1031 / \$ 02.50$ 
Removing the assumption $q(t) \geqslant 0$ from Waltman's theorem, Legatos and Kartsatos [7] proved the following result:

THEOREM B. In addition to (4) assume that

$$
\int_{1}^{\infty} \frac{d t}{f(t)}<\infty, \quad \int_{-1}^{-\infty} \frac{d t}{f(t)}<\infty
$$

Then every solution of (3) is either oscillatory or tends monotonically to zero as $t \rightarrow \infty$.

Under the same assumptions of Theorem B, Travis [10, Theorem 2.1] proved all solutions of (3) to be oscillatory.

Recently, the present author [13] gave a new criterion for the oscillation of (3) by removing the condition (5) and using the $n$th primitive of the coefficient $q(t)$ for some integer $n \geqslant 3$.

The purpose of this note is to establish some new oscillation criteria for the following more general nonlinear second order differential equation with damped term

$$
y^{\prime \prime}(t)+p(t) y^{\prime}(t)+q(t) f(y(t))=0
$$

where $p, q \in C\left[t_{0}, \infty\right), f \in C(R), y f(y)>0$ for $y \neq 0$.

Results for (6) with nonlinear damping have been obtained by Baker [2], Bobisud [3], Butler [4] and Pintér [9].

By a solution of (6) at $t_{0} \geqslant 0$ is meant a function $y:\left[t_{0}, t_{1}\right) \rightarrow R, t_{0}<t_{1}$, which satisfies (6) for all $t \in\left[t_{0}, t_{1}\right)$. We assume the existence of solutions of (6) at $t_{0}$ for every $t_{0} \geqslant 0$. A solution $y(t)$ of (6) at $t_{0}$ is said to be continuable if $y(t)$ exists for all $t \geqslant t_{0}$. A continuable solution $y(t)$ of $(6)$ is called oscillatory if $y(t)$ has zeros for arbitrarily large $t$ and nonoscillatory if there exists $t^{*} \geqslant 0$ such that $y(t) \neq 0$ for all $t \geqslant t^{*}$.

2. $q(t)$ is not assumed positive. In this section, we treat the case that $q(t)$ is not assumed positive. At first, we give a new criterion for the oscillation of (6).

THEOREM 1. Let $f^{\prime}(y)$ exist and $f^{\prime}(y) \geqslant k>0$ for $y \in R^{\prime} \equiv R-\{0\}$. If

$\left(\mathrm{C}_{1}\right) \quad \cdot \limsup _{t \rightarrow \infty} \frac{1}{t^{n-1}} \int_{t_{0}}^{t}(t-u)^{n-1} u q(u) d u=\infty$,

(C) $\quad \lim _{t \rightarrow \infty} \frac{1}{t^{n-1}} \int_{t_{0}}^{t} u\left[(t-u)\left(p(u)-\frac{1}{u}\right)+n-1\right]^{2}(t-u)^{n-3} d u<\infty$

for some integer $n \geqslant 3$, then every solution of (6) is oscillatory.

Proof. Let $y(t)$ be a nonoscillatory solution of (6) which, without loss of generality, we may assume $y(t) \neq 0$ for $t \geqslant t_{0}$. Define

$$
w(t)=\frac{t y^{\prime}(t)}{f(y(t))} \text {. }
$$

Then $w(t)$ satisfies

$$
w^{\prime}(t)-\frac{w(t)}{t}+p(t) w(t)+q(t) t+w^{2}(t) \frac{f^{\prime}(y(t))}{t}=0 .
$$


This and $f^{\prime}(y) \geqslant k>0$ for $y \neq 0$ imply

$$
w^{\prime}(t)+\left[p(t)-\frac{1}{t}\right] w(t)+q(t) t+\frac{k}{t} w^{2}(t) \leqslant 0 .
$$

Thus

$$
\begin{aligned}
\int_{t_{0}}^{t}(t-u)^{n-1} w^{\prime}(u) d u & +\int_{t_{0}}^{t}(t-u)^{n-1}\left(k u^{-1} w^{2}(u)+\left[p(u)-u^{-1}\right] w(u)\right) d u \\
& +\int_{t_{0}}^{t}(t-u)^{n-1} u q(u) d u \leqslant 0
\end{aligned}
$$

Since

$$
\int_{t_{0}}^{t}(t-u)^{n-1} w^{\prime}(u) d u=(n-1) \int_{t_{0}}^{t}(t-u)^{n-2} w(u) d u-w\left(t_{0}\right)\left(t-t_{0}\right)^{n-1},
$$

we get

$$
\begin{aligned}
& \frac{1}{t^{n-1}} \int_{t_{0}}^{t}(t-u)^{n-1} u q(u) d u \leqslant w\left(t_{0}\right)\left(\frac{t-t_{0}}{t}\right)^{n-1} \\
& \quad-t^{1-n} \int_{t_{0}}^{t}\left(k u^{-1}(t-u)^{n-1} w^{2}(u)+\left[(t-u)^{n-1}\left(p(u)-u^{-1}\right)\right.\right. \\
& = \\
& \left.\left.\quad w\left(t_{0}\right)\left(\frac{t-t_{0}}{t}\right)^{n-1}+\left(4 k t^{n-1}\right)^{-1} \quad+(n-1)(t-u)^{n-2}\right] w(u)\right) d u \\
& \quad \cdot \int_{t_{0}}^{t}\left[(t-u)\left(p(u)-u^{-1}\right)+n-1\right]^{2} u(t-u)^{n-3} d u \\
& \quad-t^{1-n} \int_{t_{0}}^{t}\left[\left(k u^{-1}(t-u)^{n-1}\right)^{1 / 2} w(u)\right. \\
& \left.\quad+\frac{(t-u)\left(p(u)-u^{-1}\right)+n-1}{2 k^{1 / 2}} u^{1 / 2}(t-u)^{(n-3) / 2}\right]^{2} d u \\
& \leqslant w\left(t_{0}\right)+\left(4 k t^{n-1}\right)^{-1} \int_{t_{0}}^{t}\left[(t-u)\left(p(u)-u^{-1}\right)+n-1\right]^{2} u(t-u)^{n-3} d u \\
& \rightarrow w\left(t_{0}\right)+M \equiv \text { a finite number, }
\end{aligned}
$$

as $t \rightarrow \infty$ by $\left(\mathrm{C}_{2}\right)$, which contradicts condition $\left(\mathrm{C}_{1}\right)$. This proves our theorem.

REMARK 1. It follows from $\left(\mathrm{C}_{2}\right)$ that $p(t) \neq 0$ in Theorem 1 , in which $p(t)$ can be thought of as a small perturbation of $1 / t$.

EXAMPLE 1. Consider the equation

$$
y^{\prime \prime}(t)+\frac{1}{t} y^{\prime}(t)+\frac{1}{t^{2}} y(t)=0, \quad t \geqslant 1 .
$$

All conditions of Theorem 1 are satisfied for $n=3$. Hence all solutions of equation (E) are oscillatory, whereas none of the known criteria [1], [7], [8], [10], [11] can obtain this result. One such solution of equation $(\mathrm{E})$ is $y(t)=\sin (\ln t)$. 
The following theorem extends the results of [1], [6], [12], [13] to equation (6) and consequently improves the results in [7], [8], [10].

THEOREM 2. Let $f^{\prime}(y)$ exist and $f^{\prime}(y) \geqslant k>0$ for $y \in R^{\prime}$. If

$$
\begin{gathered}
\limsup _{t \rightarrow \infty} \frac{1}{t^{n-1}} \int_{t_{0}}^{t}(t-u)^{n-1} q(u) d u=\infty, \\
\lim _{t \rightarrow \infty} \frac{1}{t^{n-1}} \int_{t_{0}}^{t}[(t-u) p(u)+n-1]^{2}(t-u)^{n-3} d u<\infty
\end{gathered}
$$

for some integer $n \geqslant 3$, then every solution of (6) is oscillatory.

Proof. Let $y(t)$ be a nonoscillatory solution of (6), which without loss of generality, we may assume $y(t) \neq 0$ for $t \geqslant t_{0}$. Letting $w(t)=y^{\prime}(t) / f(y(t))$, we have

$$
w^{\prime}(t)+w^{2}(t) f^{\prime}(y(t))+p(t) w(t)+q(t)=0 .
$$

Thus

$$
w^{\prime}(t)+k w^{2}(t)+p(t) w(t)+q(t) \leqslant 0 .
$$

Hence

$$
\begin{aligned}
\int_{t_{0}}^{t}(t-u)^{n-1} w^{\prime}(u) d u & +\int_{t_{0}}^{t}(t-u)^{n-1}\left[k w^{2}(u)+p(u) w(u)\right] d u \\
& +\int_{t_{0}}^{t}(t-u)^{n-1} q(u) d u \leqslant 0
\end{aligned}
$$

As in the proof of Theorem 1, we have

$$
\begin{aligned}
& t^{1-n} \int_{t_{0}}^{t}(t-u)^{n-1} q(u) d u \leqslant w\left(t_{0}\right)\left(\frac{t-t_{0}}{t}\right)^{n-1} \\
& -t^{1-n} \int_{t_{0}}^{t}\left[k^{1 / 2}(t-u)^{(n-1) / 2} w(u)\right. \\
& \left.\quad+\frac{(t-u) p(u)+n-1}{2 k^{1 / 2}}(t-u)^{(n-3) / 2}\right]^{2} d u \\
& +\left(4 k t^{n-1}\right)^{-1} \int_{t_{0}}^{t}[(t-u) p(u)+n-1]^{2}(t-u)^{n-3} d u \\
& \rightarrow w\left(t_{0}\right)+M_{0} \equiv \text { a finite number, }
\end{aligned}
$$

as $t \rightarrow \infty$, which contradicts condition $\left(\mathrm{C}_{3}\right)$. Thus our proof is complete.

REMARK 2. It follows from $\left(C_{4}\right)$ that $p(t)$ may be equal to zero in Theorem 2 , in which $p(t)$ can be thought of as a small perturbation of 0 .

Taking $p(t)=0$ in equation (6), we see easily that condition $\left(C_{4}\right)$ can be removed and we have the following result:

COROLlaRY 1 [13]. Let $f^{\prime}(y)$ exist and $f^{\prime}(y) \geqslant k>0$ for $y \in R^{\prime}$. If $\left(\mathrm{C}_{3}\right)$ holds, then every solution of (3) is oscillatory.

REMARK 3. Let $f(y)=y$ in Corollary 1. If (2) holds, then $\left(\mathrm{C}_{3}\right)$ holds for $n=3$. Thus Wintner's result [12] is a special case of Corollary 1. 
EXAMPLE 2. Consider the equation

$$
y^{\prime \prime}(t)+\frac{1}{2 t} y^{\prime}(t)+\frac{1}{4 t} y(t)=0, \quad t \geqslant 1 .
$$

All conditions of Theorem 2 are satisfied for $n=3$. Hence every solution of equation (F) is oscillatory, whereas none of the known criteria [7], [8], [10] can obtain this result. One such solution of equation (F) is $y(t)=8 \sin \sqrt{t}$.

3. $q(t)$ is eventually nonnegative. In this section, we discuss the case that $q(t)$ is eventually nonnegative and $f(y)$ is not required to be differentiable.

THEOREM 3. Let $q(t) \geqslant 0$ and $f(y) / y \geqslant k>0$ for $y \neq 0$. If $\left(\mathrm{C}_{3}\right)$ and $\left(\mathrm{C}_{4}\right)$ holds, then every solution of $(6)$ is oscillatory.

Proof. Assume that $y(t)$ is a nonoscillatory solution of (6). Letting $w(t)=y^{\prime}(t) / y(t)$, we have

$$
w^{\prime}(t)+w^{2}(t)+p(t) w(t)+q(t) \frac{f(y(t))}{y(t)}=0 .
$$

Hence

$$
w^{\prime}(t)+w^{2}(t)+p(t) w(t)+k q(t) \leqslant 0
$$

Thus

$$
\begin{aligned}
\int_{t_{0}}^{t}(t-u)^{n-1} w^{\prime}(u) d u & +\int_{t_{0}}^{t}(t-u)^{n-1}\left[w^{2}(u)+p(u) w(u)\right] d u \\
& +k \int_{t_{0}}^{t}(t-u)^{n-1} q(u) d u \leqslant 0
\end{aligned}
$$

As in the proof of Theorem 1, we have

$$
\begin{aligned}
\frac{k}{t^{n-1}} \int_{t_{0}}^{t}(t-u)^{n-1} q(u) d u \leqslant w\left(t_{0}\right)\left(\frac{t-t_{0}}{t}\right)^{n-1} \\
\quad-t^{1-n} \int_{t_{0}}^{t}\left[(t-u)^{(n-1) / 2} w(u)+\frac{(t-u) p(u)+n-1}{2}(t-u)^{(n-3) / 2}\right]^{2} d u \\
\quad+4^{-1} t^{1-n} \int_{t_{0}}^{t}[(t-u) p(u)+n-1]^{2}(t-u)^{n-3} d u \\
\rightarrow w\left(t_{0}\right)+L \equiv \text { a finite number, }
\end{aligned}
$$

as $t \rightarrow \infty$, which contradicts condition $\left(\mathrm{C}_{3}\right)$. Thus our proof is complete.

Corollary 2. Let $q(t) \geqslant 0, f(y) / y \geqslant k>0$ for $y \neq 0$. If $\left(\mathrm{C}_{3}\right)$ holds, then every solution of (3) is oscillatory.

REMARK 4. The theorems and corollaries obtained in this note apply even when the weaker condition

$$
\int_{a}^{\infty} \frac{d y}{f(y)}<\infty, \quad \int_{-a}^{-\infty} \frac{d y}{f(y)}<\infty
$$

fails for each $a>0$; for example, $f(y)=y$ in equations (E) and (F). 
ACKNOWLEDGement. The author wishes to thank the referee for his helpful comments.

\section{REFERENCES}

1. F. V. Atkinson, On second order nonlinear oscillations, Pacific J. Math. 5 (1955), 643-647.

2. J. Baker, Oscillation theorems for a second order damped nonlinear differential equation, SIAM J. Appl. Math. 25 (1973), 37-40.

3. L. Bobisud, Oscillation of solutions of damped nonlinear equations, SIAM J. Appl. Math. 19 (1970), $601-606$.

4. G. J. Butler, The oscillatory behavior of a second order nonlinear differential equation with damping, J. Math. Anal. Appl. 57 (1977), 273-289.

5. P. Hartman, On nonoscillatory linear differential equations of second order, Amer. J. Math. 74 (1952), 389-400.

6. I. V. Kamenev, Integral criterion for oscillation of linear differential equation of second order, Mat. Zametki 23 (1978), 249-251.

7. G. G. Legatos and A. G. Kartsatos, Further results on the oscillation of solutions of second order equations, Math. Japon. 14 (1968), 67-73.

8. M. Naito, Oscillation criteria for a second order differential equation with a damping term, Hiroshima Math. J. 4 (1974), 285-291.

9. L. Pinter, Oscillatory properties of certain second order differential equations, Colloq. Math. Soc. Janos Bolyai 15 (1975), 347-354.

10. C. C. Travis, A note on second order nonlinear oscillation, Math. Japon. 18 (1973), 261-264.

11. P. Waltman, Oscillations of solutions of a nonlinear equation, SIAM Rev. 5 (1963), 128-130.

12. A. Wintner, A criterion of oscillatory stability, Quart. Appl. Math. 7 (1969), 115-117.

13. C. C. Yeh, An oscillation criterion for second order nonlinear differential equations with functional arguments, J. Math. Anal. Appl. 76 (1980), 72-76.

Department of Mathematics, Central University, Chung-Li, Taiwan, Republic of China 